\title{
Exchange-Correlation Energy from Pairing Matrix Fluctuation and the Particle-Particle Random Phase Approximation
}

\author{
Helen van Aggelen \\ Ghent University, Department of Inorganic and Physical Chemistry, 9000 Ghent, Belgium and \\ Duke University, Department of Chemistry, NC 27708, U.S. \\ Yang Yang \\ Duke University, Department of Chemistry, NC 27708, U.S. \\ Weitao Yang \\ Duke University, Department of Chemistry and Department of Physics, NC 27708, U.S.
}

(Dated: September 8, 2013)

\begin{abstract}
We formulate an adiabatic connection for the exchange-correlation energy in terms of pairing matrix fluctuation. This connection opens new channels for density functional approximations based on pairing interactions. Even the simplest approximation to the pairing matrix fluctuation, the particle-particle Random Phase Approximation (pp-RPA), has some highly desirable properties. It has no delocalization error with a nearly linear energy behavior for systems with fractional charges, describes van der Waals interactions similarly and thermodynamic properties significantly better than particle-hole RPA, and eliminates static correlation error for single-bond systems. Most significantly, the pp-RPA is the first known functional that has an explicit and closed-form dependence on the occupied and unoccupied orbitals and captures the energy derivative discontinuity in strongly correlated systems. These findings illlustrate the potential of including pairing interactions within a density functional framework.
\end{abstract}

The desire for systematic progress in Density Functional Approximations (DFA) has drawn attention to functionals rooted in many-body perturbation theory 1 3], the most popular of which is the Random Phase Approximation (RPA). The RPA originated in nuclear many-body theory in the 1950s [4, [5 but recently found new applications formulated as a DFA of occupied and virtual orbitals [6]. The DFA perspective is justified by the adiabatic connection fluctuation dissipation (ACFD) theorem[7, which establishes a fundamental connection between DFT and many-body perturbation theory. It formulates the exchange-correlation energy in terms of the polarization propagator, for which the RPA provides an approximation. The RPA overcomes some persistent problems of classical DFA functionals. In contrast to most classical DFA functionals, it describes static correlation correctly and thus dissociates, for instance, $\mathrm{H}_{2}$ correctly [8]; it captures long-range interactions adequately and is applicable to systems with vanishing gap [9]. These desirable features have been the incentive to construct more efficient algorithms, such that large-scale applications are now feasible [10]. Nonetheless, the RPA still faces some major theoretical challenges: it violates the Pauli principle, which leads to a large delocalization error, as demonstrated in the dissociation of $\mathrm{H}_{2}^{+}$ and other molecules 11. The Second Order Screened Exchange (SOSEX) 12] corrects this problem [13, but fails in cases of static correlation such as dissociating $\mathrm{H}_{2}$.

All of the RPA-related DFA methods are based on particle-hole (ph) interactions [9, 10, 14, 15. However, the second-order Green's function naturally leads to an- other channel of interactions: particle-particle (pp) and hole-hole (hh) interactions [16]. The present work establishes an adiabatic connection[17, 18, for the exchangecorrelation energy in terms of the dynamic paring matrix fluctuation or particle-particle Green function, parallel to the ACFD theorem in terms of the density fluctuation or polarization propagator. Like the ACFD theorem, it is in principle exact, but requires the particle-particle Green function as a function of the interaction strength. The pp-RPA, a Random Phase Approximation in the pp and hh correlation channels, provides an approximation to the $\lambda$ - dependence of the Green function that leads to a simple closed expression for the exchange-correlation energy. In this paper we therefore explore the pp-RPA as a DFA functional, based on the adiabatic connection we formulate, to illustrate the potential of using pairing interactions in DFA. Despite its close relationship to the ph-RPA, particle-particle interactions have received limited attention only in spectral calculations [19, but not as a DFA for ground state energies. The theoretical framework underlying the pp-RPA is very similar to that of ph-RPA, but its features as a DFA functional are quite different, as we will illustrate with applications to molecular dissociation and thermodynamical properties.

The exact exchange-correlation energy in Kohn-Sham DFT (KS-DFT) can be related to paring matrix fluctuation $\overline{\mathbf{K}}(E)$ (or the particle-particle Green function $\mathbf{K}(E)$ ) in many-body perturbation theory via the adiabatic connection. The pairing matrix fluctuation $\overline{\mathbf{K}}\left(t-t^{\prime}\right)$ describes the response of the pairing matrix $\kappa_{i j}(t)=$ $\left\langle\Psi_{0}^{N}\left|a_{H_{i}}(t) a_{H_{j}}(t)\right| \Psi_{0}^{N}\right\rangle$ to a perturbation in the form of 
a pairing field, $\hat{F}\left(t^{\prime}\right)=f_{k l} a_{H_{l}}^{\dagger}\left(t^{\prime}\right) a_{H_{k}}^{\dagger}\left(t^{\prime}\right) \theta\left(t^{\prime}\right)$. The operators $a_{H_{i}}^{\dagger}(t)$ are the creation operators in the Heisenberg picture, $a_{H_{i}}^{\dagger}(t)=e^{\frac{i}{\hbar}(\hat{H}-\nu \hat{N}) t} a_{i}^{\dagger} e^{\frac{-i}{\hbar}(\hat{H}-\nu \hat{N}) t}$ and the term $-\nu \hat{N}$, with $\nu$ the chemical potential, is added to the Hamiltonian such that the $N$-electron state is the minimum under the total Hamiltonian $\hat{H}-\nu \hat{N}$ when the particle number is allowed to change. In the energy domain, $\overline{\mathbf{K}}(E)$ has the form

$$
\begin{aligned}
\bar{K}(E)_{i j k l} & =\sum_{n} \frac{\left\langle\Psi_{0}^{N}\left|a_{i} a_{j}\right| \Psi_{n}^{N+2}\right\rangle\left\langle\Psi_{n}^{N+2}\left|a_{l}^{+} a_{k}^{+}\right| \Psi_{0}^{N}\right\rangle}{E-\omega_{n}^{N+2}+i \eta} \\
& -\sum_{n} \frac{\left\langle\Psi_{0}^{N}\left|a_{l}^{+} a_{k}^{+}\right| \Psi_{n}^{N-2}\right\rangle\left\langle\Psi_{n}^{N-2}\left|a_{i} a_{j}\right| \Psi_{0}^{N}\right\rangle}{E-\omega_{n}^{N-2}+i \eta}
\end{aligned}
$$

and therefore contains information on the 2-electron addition and removal energies $\omega_{n}^{N+2}$ and $\omega_{n}^{N-2}$ and the corresponding transition amplitudes. Moreover, these response functions not only provide information on the $N \pm 2$ electron excited states, they also indirectly determine ground state properties. The ground state correlation energy can be formulated in terms of the pairing matrix fluctuation (or, equivalently, the pp-Green function) through the adiabatic connection:

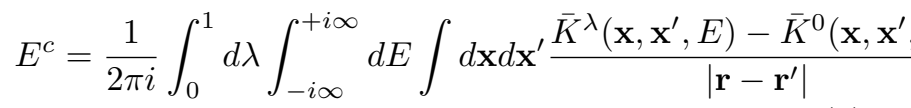

Since the exchange energy is the exact exchange, we focus on the correlation energy. Further background and full derivations are presented in sections $1 \mathrm{~A}-1 \mathrm{C}$ of the supplementary material, ref. ([20]). This formula can be considered the pairing interaction counterpart of the ACFD theorem. Like the ACFD theorem, formula (1) is in principle exact, but requires an approximation to compute the pairing matrix fluctuation $\overline{\mathbf{K}}^{\lambda}$. The simplest approximation to the pairing matrix fluctuation is the particle-particle RPA. In this work, we will focus on the particle-particle RPA to illustrate the potential of including pairing interactions in a DFT framework.

The pp-RPA approximates the dynamic pairing matrix fluctuation $\overline{\mathbf{K}}^{\lambda}$ in terms of its non-interacting counterpart $\overline{\mathbf{K}}^{0}$

$$
\overline{\mathbf{K}}^{\lambda}=\overline{\mathbf{K}}^{0}+\lambda \overline{\mathbf{K}}^{0} \mathbf{V} \overline{\mathbf{K}}^{\lambda},
$$

where the Coulomb interaction is $V_{a b c d}=$ $\langle a b \| c d\rangle=\langle a b \mid c d\rangle-\langle b a \mid c d\rangle$, and $\langle a b \mid c d\rangle=$ $\int \phi_{a}^{*}(\mathbf{x}) \phi_{b}^{*}\left(\mathbf{x}^{\prime}\right) \frac{1}{\left|\mathbf{r}-\mathbf{r}^{\prime}\right|} \phi_{c}(\mathbf{x}) \phi_{d}\left(\mathbf{x}^{\prime}\right) d \mathbf{x} d \mathbf{x}^{\prime} . \quad$ Under this approximation, the integration over the interaction strength $\lambda$ in Eq. (1) can be carried out analytically. The resulting expression for the correlation energy in terms of the non-interacting Green function $\mathbf{K}^{0}$ is equivalent to the sum of all ladder diagrams in the context of many-body perturbation theory [16]

$$
\begin{aligned}
E^{c} & \left.=\frac{-1}{2 \pi i} \sum_{n=2} \frac{1}{n} \int_{-i \infty}^{+i \infty} \operatorname{tr}\left[\overline{\mathbf{K}}^{0}(E)\right) \mathbf{V}\right]^{n} d E \\
& =\frac{1}{2 \pi i} \int_{-i \infty}^{+i \infty} \operatorname{tr}\left[\ln \left(\mathbf{I}-\overline{\mathbf{K}}^{0}(E) \mathbf{V}\right)+\overline{\mathbf{K}}^{0}(E) \mathbf{V}\right]
\end{aligned}
$$

The pairing matrix fluctuation $\overline{\mathbf{K}}(E)$ is antisymmetrical under particle exchange, so Eq. (1)-22) are formulated in an antisymmetrical basis, which includes only ordered two-particle indices. While the correlation energy can be computed directly from Eq. 22, it can also be cast in terms of the solution to a generalized eigenvalue problem (see Eq. (11) of ref. $([20])$ ), which requires $O\left(N_{h}^{2} N_{p}^{4}\right)$ operations to evaluate,

$$
\begin{aligned}
& \sum_{c<d}\left(\langle a b \| c d\rangle+\delta_{a c} \delta_{b d} \omega_{a b}^{0}\right) X_{c d}^{n}+\sum_{i<j}\langle a b \| i j\rangle Y_{i j}^{n}=\omega_{n} X_{a b}^{n} \\
& \sum_{a<b}\langle i j \| a b\rangle X_{a b}^{n}+\sum_{k<l}\left(\langle i j \| k l\rangle-\delta_{i k} \delta_{j l} \omega_{i j}^{0}\right) Y_{k l}^{n}=-\omega_{n} Y_{i j}^{n}
\end{aligned}
$$

where $\omega_{a b}^{0}=\epsilon_{a}+\epsilon_{b}-2 \nu$. This eigenvalue problem is then solved for the pp-RPA eigenvectors $\mathbf{X}^{\mathbf{n}}, \mathbf{Y}^{\mathbf{n}}$

, Eind their corresponding eigenvalues $\omega_{n}$. The generalized eigenvalues $\omega_{n}$ have a clear physical meaning: they are either positive 2-electron addition energies, $\omega_{n}^{N+2}=$ $E_{n}^{N+2}-E_{0}^{N}-2 \nu$, or negative 2-electron removal energies, $\omega_{n}^{N-2}=E_{0}^{N}-E_{n}^{N-2}-2 \nu$. The eigenvectors are the corresponding amplitudes, $X_{a b}^{n}=\left\langle\Psi_{0}^{N}\left|a_{a} a_{b}\right| \Psi_{n}^{N+2}\right\rangle$ and $Y_{i j}^{n}=$ $\left\langle\Psi_{0}^{N}\left|a_{i} a_{j}\right| \Psi_{n}^{N+2}\right\rangle$ when $\omega_{n}>0 ; X_{a b}^{n}=\left\langle\Psi_{0}^{N}\left|a_{b}^{+} a_{a}^{+}\right| \Psi_{n}^{N-2}\right\rangle$ and $Y_{i j}^{n}=\left\langle\Psi_{0}^{N}\left|a_{j}^{+} a_{i}^{+}\right| \Psi_{n}^{N-2}\right\rangle$ when $\omega_{n}<0$.

The pp-RPA correlation energy can be reformulated in terms of the solution to this generalized eigenvalue system (see section $1 \mathrm{C}$ of ref. ([20])):

$$
E^{c}=\sum_{n} \omega_{n}^{N+2}-\sum_{a<b}\left(\epsilon_{a}+\epsilon_{b}-2 \nu+\langle a b \| a b\rangle\right)
$$

where the summation over $n$ runs over all 2-electron addition energies. Since Eq. (3) depends only on the orthonormal set of orbitals $\left\{\phi_{i}\right\}$ and their occupations $n_{i}$, the correlation energy can be viewed as a functional $E\left[\left\{\phi_{i}\right\}, n_{i}\right]$. The total pp-RPA energy expression combines the HF-energy functional with the pp-RPA correlation energy, Eq. (4).

The density functional perspective on the pp-RPA raises some prominent questions: how does the pp-RPA behave for systems with fractional spins or charges, which present a major challenge for DFA? [1, 22]. Most approximate density functionals give physically incorrect properties for systems that arise from an ensemble, such as molecule fragments with fractional electron numbers or 
spins. Such systems naturally arise for instance as the dissociation products of a molecule. While the molecule as a whole has integer electron number and (half) integer spin, each of its dissociation products may have a fractional electron number or spin. The exact conditions on density functionals for fractional charges [21, 23], fractional spins 24], and their combination 25] are now known.

The performance of density functionals for systems with fractional occupation number has therefore become an important criterion in the development of DFA. The behavior of the pp-RPA for such systems can be quantified by taking the fractional orbital occupations into account explicitly in the pp-RPA equations (section $1 \mathrm{E}$ of ref. $(20])$ ), following previous work extending other DFAs to fractionals [11, 24.

We computed the Kohn-Sham (KS) reference wavefunction with Gaussian03 26] for the systems with integer electron number and with the QM4D package for systems with fractional electron number or spin [27. For the subsequent pp-RPA calculation, we used our implementation, which diagonalizes the pp-RPA matrix. Since the diagonalization is computationally expensive, we used a cc-pVDZ basis set for all calculations, except for the Ar and Ne atoms, for which we used an aug-cc-pVDZ (FC) basis set. For the calculations on thermodynamical properties, we used a cc-pVTZ basis set limited to F-functions because the pp-RPA energy converges slowly with the basis set size (Fig. 12 of ref. ([20])) and geometries from the G2 test set 28. Accurate potential energy functions for the dimers of the noble gases have been taken from the work of Ogilvie et al. [29, 30] and the MRCI potential energy function for the $\mathrm{N}_{2}$ in the cc-pVDZ basis set has been taken from previous work [31.

The pp-RPA has negligible delocalization error and static correlation error and thus produces the correct dissociation limit for $\mathrm{H}_{2}$ and $\mathrm{H}_{2}^{+}$. The $\mathrm{H}_{2}$ and $\mathrm{H}_{2}^{+}$molecules are paradigmatic examples of challenges for DFA 22, because few DFA functionals give the correct dissociation limit for both $\mathrm{H}_{2}$ and $\mathrm{H}_{2}^{+}$. The ph-RPA dissociates $\mathrm{H}_{2}$ correctly, but produces a huge delocalization error for $\mathrm{H}_{2}^{+}$ 11. The pp-RPA, however, gives the correct dissociation limit for $\mathrm{H}_{2}$ and $\mathrm{H}_{2}^{+}$, although the potential energy curve of $\mathrm{H}_{2}$ has an unphysical local maximum around $10 \AA$ (Figs. 1 and Fig. 2 of ref. ([20])). While the dissociation of $\mathrm{H}_{2}^{+}$is described correctly by construction in pp-RPA - the pp-RPA energy reduces to the HF functional for a one-electron system - it also gives a good dissociation profile for $\mathrm{He}_{2}^{+}$, for instance (Fig. 2). Other RPA methods have been constructed to dissociate these positively charged molecules correctly, such as ph-RPA+SOSEX, which a posteriori corrects for neglecting antisymmetry in the ph-RPA. However, RPA+SOSEX gives a much too high dissociation limit for $\mathrm{H}_{2} 13$.

The pp-RPA satisfies the Hydrogen Test Set (1]): it has no delocalization error for $\mathrm{H}_{2}^{+}$and almost no static correlation error for $\mathrm{H}_{2}$ because it has a nearly physically correct energy profile for the $\mathrm{H}$ atom with fractional charges and fractional spins. Describing both cases correctly requires that the functional has constant energy for all spin projections between 0 and 1 [24, 25, and that it has a linear energy profile for electron numbers between 0 and 1 [23. Most DFA functionals do not have these features, which results in static correlation errors and/or delocalization errors. The ph-RPA, for instance, has a nearly constant energy for different spin projections in the $\mathrm{H}$ atom, but has a significant delocalization error for fractional electron numbers [11] (Figs. 4 and 5 of ref. $([20]))$. It thus describes the dissociation of $\mathrm{H}_{2}$ correctly but gives a much too low dissociation limit for $\mathrm{H}_{2}^{+}$. The pp-RPA not only has a nearly constant energy for different spin projections of the $\mathrm{H}$ atom but also has a nearly linear energy between electron numbers of 0 and 1 (Fig. 4 of ref. $([20]))$. These properties ensure that it gives the right dissociation limit for $\mathrm{H}_{2}$ and $\mathrm{H}_{2}^{+}$.

Most significantly, the pp-RPA captures the energy derivative discontinuity for strongly correlated systems (SCS) at integer electron numbers. Traditional DFA functionals have a smooth dependence on the occupied orbitals and cannot capture the required derivative discontinuities for SCS at integer electron number 24, 25. Even the ph-RPA energy, which is a functional of the occupied and the unoccupied orbitals, does not have a derivative discontinuity at integer electron numbers for SCS. (Figs. 4 and 5 of ref. (20)). 11 However, the pp-RPA adequately captures the energy derivative discontinuity and satisfies the flat-plane condition 25, as Fig. 4 of ref. (20]) and Fig. 3 illustrate for the $\mathrm{H}$ atom and $\mathrm{Li}$ atom.

The pp-RPA describes the ionization energies exceptionally well, although in the present basis set the sign of some of the very small electron affinities is wrong. Finitedifference calculations on the pp-RPA chemical potential for a set of second-period atoms (table II of ref. (20])) demonstrate the superiority of the pp-RPA over the phRPA.

The pp-RPA has almost no static correlation error for single-bond systems, and gives the proper dissociation limit for ethane, for instance (Fig. 7 of ref. ([20])). However, it has a substantial error for the dissociation of $\mathrm{N}_{2}$ (Fig. 8 of ref. ([20])). Breaking multiple bonds like those in $\mathrm{N}_{2}$ within a singlet description is problematic for ppRPA because $\mathrm{N}_{2}$ dissociates into two spin-unpolarized spherical $\mathrm{N}$ atoms, which have equal fractional numbers of alpha and beta electrons distributed evenly over the three p-orbitals, and pp-RPA assigns much too low energy to these spin-unpolarized spherical atoms (Fig. 9 of ref. $([20]))$.

The pp-RPA describes van der Waals interactions to a very good extent, similar to ph-RPA and phRPA+SOSEX [13, 32. One of the main strengths of ph-RPA is its ability to capture non-covalent long-range 
interactions smoothly and seamlessly. Although the nature of the interactions is different in pp-RPA from that in ph-RPA, pp-RPA also captures the van der Waals interactions in $\mathrm{Ar}_{2}$ and NeAr well (Figs. 4 and Fig. 11 of ref. $(20]))$.

The pp-RPA performs much better than ph-RPA on the heats of formation and atomization energies for a set of small molecules. The mean absolute errors (MAE) on the heats of formation computed for a set of small molecules is $5.8 \mathrm{kcal} / \mathrm{mol}$ for the pp-RPA and 10.4 $\mathrm{kcal} / \mathrm{mol}$ - in good agreement with the results by Ren et al. [10] - for the ph-RPA (table III of ref. ([20])). The $4.6 \mathrm{kcal} / \mathrm{mol}$ difference shows that the accuracy of the heats of formation computed with pp-RPA is better than that of ph-RPA. Furthermore, a test on the whole G2 set shows that the errors in the ph-RPA heats of formation increase steadily with the number of atoms in the molecules, whereas the errors in the pp-RPA heats of formation remain nearly constant (Fig. 5).

Finally, a perturbation theory analysis (section 1D of ref. ([20])) shows that pp-PRA has the correct secondorder energy, in contrast to the ph-RPA, which contains only the direct terms of the second-order energy.

To summarize, we have shown that the exact exchangecorrelation energy can be expressed in terms of the dynamic paring matrix fluctuation via the adiabatic connection and illustrated the potential of this approach with the pp-RPA. The pp-RPA is a remarkable DFA, because it is the first functional that has an explicit and closedform dependence on the occupied and virtual orbitals and captures the derivative discontinuity of the energy at integer electron numbers for the whole range of spin polarizations in strongly correlated systems.

The pp-RPA meets the flat-plane energy requirement for systems with fractional charges and spins [25]. This flat-plane energy behavior has been actively pursued in recent years, with limited success up to now [33]. It was shown that explicit, differentiable functionals of the density or density matrix cannot capture it [11,24]. Even the inclusion of virtual orbitals in the ph-RPA does not prove helpful [11. The discontinuity in the pp-RPA energy as shown presently proves that this goal can be achieved in closed form with a functional that depends on both the occupied and unoccupied orbitals, or on the Green's function of the non-interacting (generalized) KS reference system, highlighting the path forward for development of functionals for strongly correlated systems.

At the time of the final revision of this manuscript two related manuscripts, which explore the pp-RPA for molecular calculations from the coupled-cluster perspective, were brought to our attention (arXiv:1306.6360, arXiv:1306.5638). Support from FWO-Flanders (Scientific Research Fund Flanders) (H.v.A), the Office of Naval Research (N00014-09- 0576) and the National Science Foundation (CHE-09-11119) (W.Y.) is appreciated.

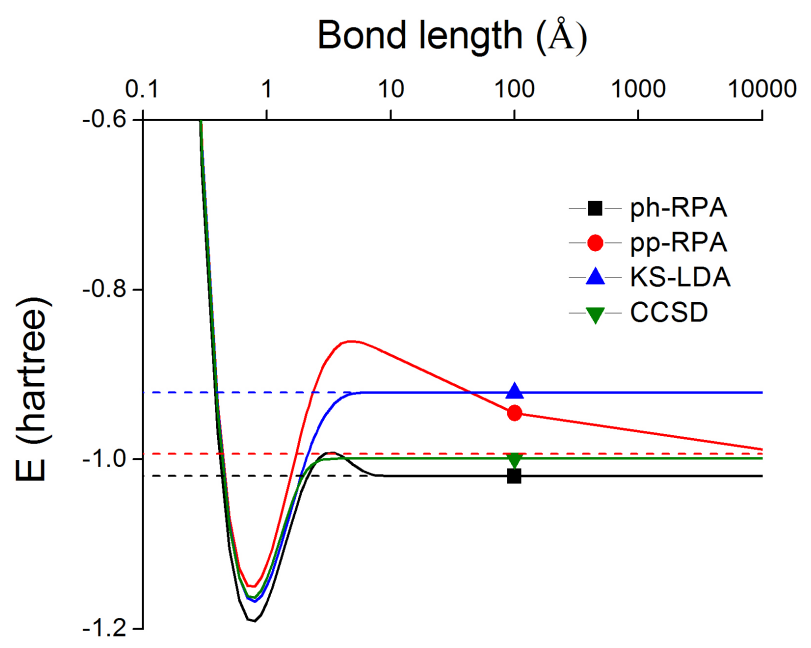

FIG. 1: (Color online) The pp-RPA(LDA) energy for the $\mathrm{H}_{2}$ molecule approaches the correct value in the dissociation limit, but has an unphysical 'bump', much more so than phRPA(LDA). The dashed lines indicate the dissociation limits from the fractional analysis of the $\mathrm{H}$ atom.

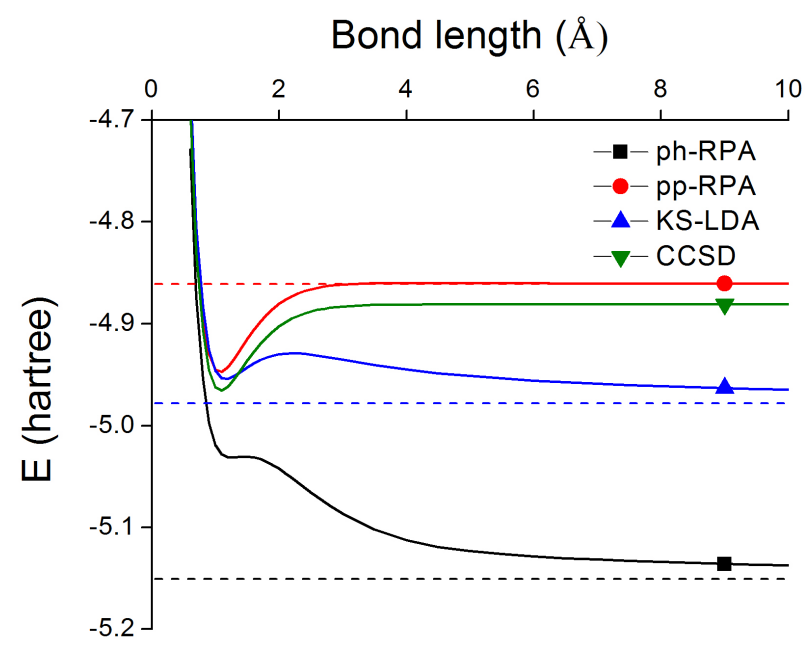

FIG. 2: (Color online) The pp-RPA(LDA) also gives a correct energy profile for $\mathrm{He}_{2}^{+}$, in contrast to ph-RPA(LDA). The dashed lines indicate the dissociation limits from the fractional analysis of the He atom. 


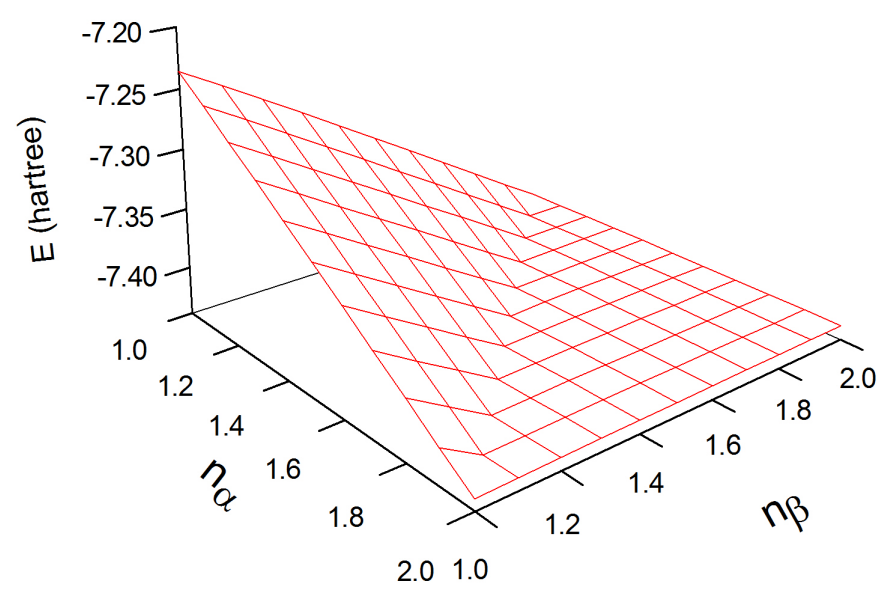

FIG. 3: (Color online) The pp-RPA(LDA) energy for the Li atom is a nearly constant function of the fractional spin projection and a nearly linear function of the fractional electron number. Like the exact functional, its derivative has a discontinuity at $\mathrm{N}=3$.

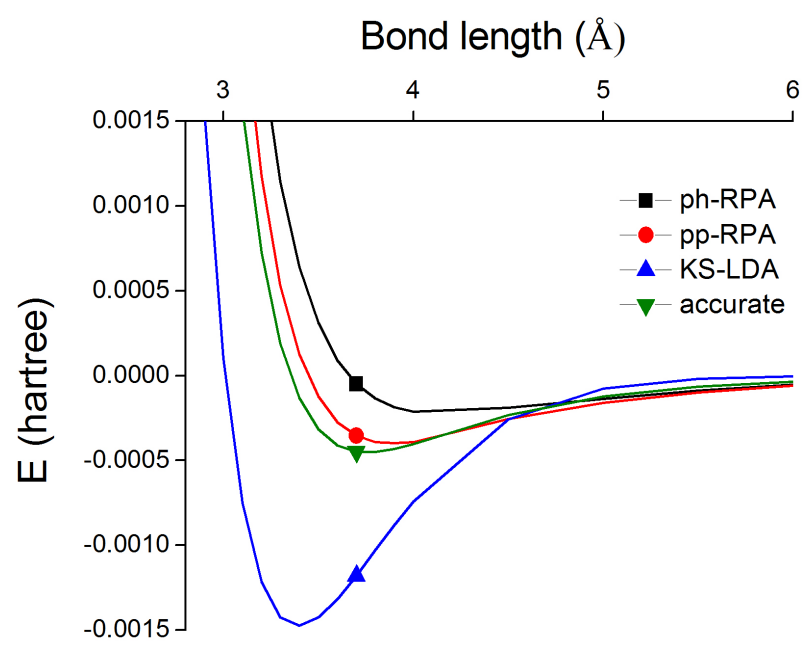

FIG. 4: (Color online) The ph-RPA(LDA) and pp-RPA(LDA) both describe the van der Waals interactions in the Ar dimer well.

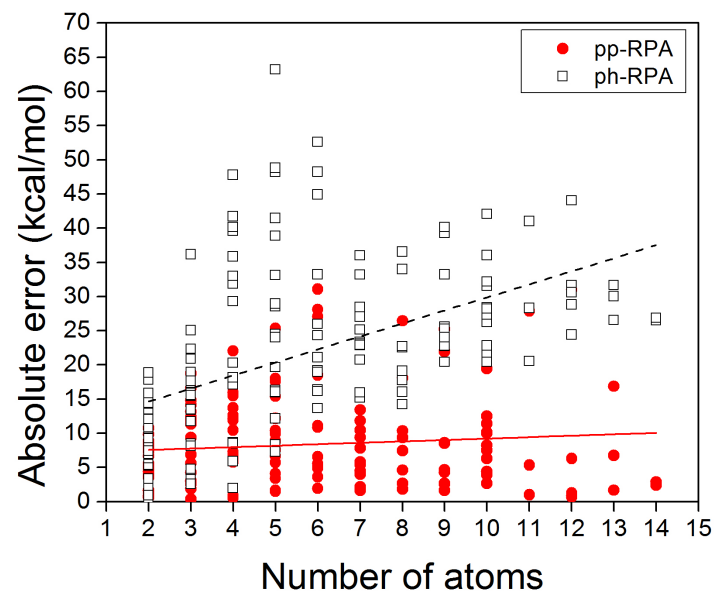

FIG. 5: (Color online) The ph-RPA(PBE) enthalpies of formation for the molecules in the G2/97 database show a steadily increasing error with the number of atoms, with a MAE of $22.7 \mathrm{kcal} / \mathrm{mol}$ whereas the pp-RPA(PBE) enthalpies show nearly constant errors with the number of atoms, with a MAE of $8.3 \mathrm{kcal} / \mathrm{mol}$. 
[1] A. J. Cohen, P. Mori-Sanchez, and W. T. Yang, Chem Rev 112, 289 (2012).

[2] K. Burke, Journal of Chemical Physics 136 (2012).

[3] G. Onida, L. Reining, and A. Rubio, Reviews of Modern Physics 74, 601 (2002).

[4] D. Bohm and D. Pines, Phys. Rev. 82, 625 (1951).

[5] P. Ring and P. Schuck, The Nuclear Many-Body Problem, Texts and Monographs in Physics (Springer-Verlag, 1980).

[6] M. Fuchs and X. Gonze, Physical Review B 65, 235109 (2002).

[7] D. C. Langreth and J. P. Perdew, Phys. Rev. B 15, 2884 (1977).

[8] A. Heßelmann and A. Görling, Phys. Rev. Lett. 106, 093001 (2011).

[9] H. Eshuis, J. E. Bates, and F. Furche, Theoretical Chemistry Accounts 131 (2012).

[10] X. Ren, P. Rinke, C. Joas, and M. Scheffler, Journal of Materials Science 47, 7447 (2012).

[11] P. Mori-Sanchez, A. J. Cohen, and W. T. Yang, Physical Review A 85 (2012).

[12] D. L. Freeman, Physical Review B 15, 5512 (1977).

[13] A. Gruneis, M. Marsman, J. Harl, L. Schimka, and G. Kresse, J Chem Phys 131, 154115 (2009).

[14] J. Toulouse, W. M. Zhu, J. G. Angyan, and A. Savin, Physical Review A 82 (2010).

[15] X. Ren, A. Tkatchenko, P. Rinke, and M. Scheffler, Phys. Rev. Lett. 106, 153003 (2011).

[16] J. P. Blaizot and G. Ripka, Quantum Theory of Finite Systems (MIT Press, 1986).

[17] O. Gunnarsson and B. I. Lundqvist, Phys. Rev. B 13, 4274 (1976).

[18] D. Langreth and J. Perdew, Solid State Communications
17, 1425 (1975).

[19] P. Romaniello, F. Bechstedt, and L. Reining, Physical Review B 85 (2012).

[20] See supplementary material, available at ..., for background and derivations.

[21] A. J. Cohen, P. Mori-Sanchez, and W. T. Yang, Physical Review B 77 (2008).

[22] A. J. Cohen, P. Mori-Sanchez, and W. Yang, Science 321, 792 (2008).

[23] J. P. Perdew, R. G. Parr, M. Levy, and J. L. Balduz, Phys Rev Lett 49, 1691 (1982).

[24] A. J. Cohen, P. Mori-Sanchez, and W. T. Yang, Journal of Chemical Physics 129 (2008).

[25] P. Mori-Sanchez, A. J. Cohen, and W. T. Yang, Phys Rev Lett 102 (2009).

[26] M. J. Frisch, G. W. Trucks, H. B. Schlegel, G. E. Scuseria, M. A. Robb, J. R. Cheeseman, J. A. Montgomery, Jr., T. Vreven, K. N. Kudin, J. C. Burant, et al., Gaussian 03, revision b.05, Gaussian Inc. (2004).

[27] $Q M 4 D$, a program for $Q M / M M$ simulations, URL http: //www.qm4d.info

[28] L. A. Curtiss, K. Raghavachari, P. C. Redfern, and J. A. Pople, Journal of Chemical Physics 106, 1063 (1997).

[29] J. F. Ogilvie and F. Y. H. Wang, Journal of Molecular Structure-Theochem 273, 277 (1992).

[30] J. Ogilvie and F. Y. H. Wang, Journal of Molecular Structure-Theochem 291, 313 (1993).

[31] H. van Aggelen, B. Verstichel, P. Bultinck, D. Van Neck, P. W. Ayers, and D. L. Cooper, Journal of Chemical Physics 132 (2010).

[32] R. M. Irelan, T. M. Henderson, and G. E. Scuseria, J Chem Phys 135, 094105 (2011).

[33] E. R. Johnson and J. Contreras-Garcia, Journal of Chemical Physics 135, 081103 (2011). 